\title{
Passivity-preserving Parameterized Model Order Reduction for PEEC Based Full Wave Analysis
}

\author{
F. Ferranti*, G. Antonini ${ }^{+}$, T. Dhaene*, L. Knockaert* \\ ${ }^{*}$ Dept. of Information Technology (INTEC), \\ Ghent University-IBBT, Sint Pietersnieuwstraat 41, 9000 Ghent, Belgium \\ +UAq EMC Laboratory, Dipartimento di Ingegneria Elettrica e dell'Informazione, \\ Università degli Studi dell'Aquila, Monteluco di Roio, 67040, L'Aquila, Italy
}

\begin{abstract}
We present a novel parameterized model order reduction technique applicable to the Partial Element Equivalent Circuit method that is able to generate parametric reduced order models, stable and passive by construction, over a user defined design space. Overall stability and passivity of the parametric reduced order model are guaranteed by an efficient and reliable combination of traditional passivity-preserving model order reduction methods and interpolation schemes based on a class of positive interpolation operators. A pertinent numerical example validates the proposed parameterized model order reduction approach.
\end{abstract}

\section{Introduction}

Nowadays, full-wave electromagnetic (EM) methods [1-3] have become fundamental analysis and design tools for a variety of complex high-speed systems. Large systems of equations are usually generated by EM methods and model order reduction (MOR) techniques are consequently utilized to reduce the resulting high model complexity and computational cost of the simulations $[4,5]$. Over the last years, the development of a reduced order model (ROM) of the EM system has become a topic of intense research. With respect to other full-wave techniques, the Partial Element Equivalent Circuit (PEEC) method is able to transform the EM system under examination into a passive RLC equivalent circuit $[2,6]$. However, inclusion of the PEEC model directly into a circuit simulator may be computationally intractable for complex structures, because the number of circuit elements can be in the tens of thousands. MOR techniques become necessary to reduce the size of a PEEC model $[4,5]$.

Optimization and design space exploration are usually performed during a typical design process that consequently requires multiple simulations for different design parameter values. Traditional MOR techniques perform model reduction only with respect to frequency and such design activities call for parameterized model order reduction (PMOR) methods that can reduce large systems of equations with respect to frequency and other design parameters of the circuit, such as geometrical layout or substrate characteristics.

Different PMOR techniques have been developed over the last years. The multiparameter moment-matching methods presented in $[7,8]$ use a subspace projection approach and guarantee the passivity. However, the resulting reduced models usually suffer from oversize when the number of moments to match is high, either because high accuracy (order) is required or because the number of parameters is large. Some PMOR techniques are based on statistical performance analysis $[9,10]$.

This paper proposes a PMOR technique applicable to the
PEEC method that provides parametric reduced order models, stable and passive by construction, over the design space of interest. It is based on the reliable and efficient combination of traditional passivity-preserving MOR methods $[4,5]$ and interpolation schemes based on a class of positive interpolation operators [11]. In [12] a parametric macromodeling method has been recently proposed that exploits the combination of rational identification and positive interpolation schemes to build parameterized macromodels, stable and passive by construction, over the design space of interest, starting from multivariate data samples of the input-output system behavior and not from system equations as in all PMOR techniques previously discussed.

This paper is organized as follows. Section II describes the modified nodal analysis (MNA) equations of the PEEC method. Section III describes the proposed PMOR method. Finally, a pertinent numerical example is presented in Section IV, validating the proposed PMOR technique.

\section{PEEC formulation}

The PEEC method [2] is based on the integral equation form of Maxwell's equations and is able to provide a circuit interpretation of the Electric Field Integral Equation, thereby allowing to handle complex problems involving both circuits and electromagnetic fields $[2,6,13,14]$.

Once the meshing process of conductors and dielectrics has been performed, $n_{i}$ volume cells where currents flow and $n_{n}$ surface cells where charge is located are generated. The resultant number of elementary cells of conductors and dielectrics is $n_{c}$ and $n_{d}$, respectively and that of electrical nodes is $n_{n}$. If the MNA approach [15] is used, an admittance representation $\mathbf{Y}(s)$ is generated with $n_{p}$ output currents $\mathbf{i}_{p}(t)$ under voltage excitation $\mathbf{v}_{p}(t)$

$$
\begin{aligned}
\mathbf{C} \frac{d \mathbf{x}(t)}{d t} & =-\mathbf{G} \mathbf{x}(t)+\mathbf{B u}(t) \\
\mathbf{i}_{p}(t) & =\mathbf{L}^{T} \mathbf{x}(t)
\end{aligned}
$$

where the global number of unknowns is $n_{u}=n_{i}+n_{d}+n_{n}+$ $n_{p}, \mathbf{x}(t) \in \Re^{n_{u} \times 1}, \mathbf{C} \in \Re^{n_{u} \times n_{u}}, \mathbf{G} \in \Re^{n_{u} \times n_{u}}$ and $\mathbf{B}=\mathbf{L}$, $\mathbf{B} \in \Re^{n_{u} \times n_{p}}$. This is an $n_{p}$-port formulation, whereby the only sources are the voltage sources at the $n_{p}$-port nodes.

2.1 Properties of PEEC formulation Stability and passivity are fundamental properties for a model that is used in a simulator that performs transient analysis. While a passive system is also stable, the reverse is not necessarily true [16]. Some properties of the real matrices $\mathbf{B}, \mathbf{L}, \mathbf{C}, \mathbf{G}$ are fundamental for the passivity (or positive-realness) [17] of the PEEC admittance matrix $\mathbf{Y}(s)=\mathbf{L}^{T}(s \mathbf{C}+\mathbf{G})^{-1} \mathbf{B}$. A first relevant property guaranteed by construction is $\mathbf{B}=\mathbf{L}$. Since the internal block 
submatrices of the matrices $\mathbf{C}, \mathbf{G}$ are symmetric nonnegative definite matrices by construction [18], it is straightforward to prove that $\mathbf{C}, \mathbf{G}$ satisfy the following properties

$$
\begin{aligned}
& \mathbf{C}=\mathbf{C}^{T} \geq 0 \\
& \mathbf{G}+\mathbf{G}^{T} \geq 0
\end{aligned}
$$

The properties of the PEEC matrices $\mathbf{B}=\mathbf{L}, \mathbf{C}=\mathbf{C}^{T} \geq$ $0, \mathbf{G}+\mathbf{G}^{T} \geq 0$ guarantee the passivity of the PEEC admittance model [19].

\section{Parameterized model order reduction}

In this section we describe a PMOR technique that is able to generate parametric reduced order models, stable and passive by construction, over a user defined design space. These parametric reduced models depend on frequency and $\mathrm{N}$ design parameters $\boldsymbol{g}=\left(g^{(1)}, \ldots, g^{(\mathrm{N})}\right)$, such as the layout features of a circuit or the substrate parameters. The different steps of the proposed PMOR method are shown by a flowchart in Fig. 1.

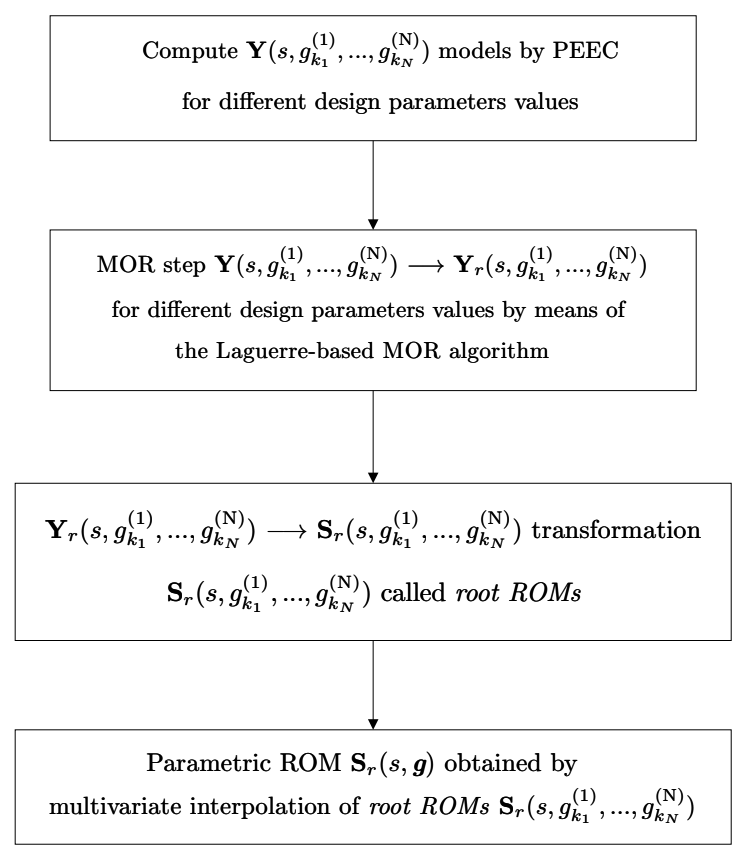

Figure 1: Flowchart of the proposed PMOR method.

3.1 Root ROMs The proposed PMOR technique starts computing a set of stable and passive reduced order models of the PEEC admittance matrix $\mathbf{Y}(s)$ using the Laguerre-based MOR algorithm [5] for different design parameters values in the design space $\left\{\boldsymbol{g}_{k}\right\}_{k=1}^{K_{t o t}}$. The $\mathbf{S}$-representation is used to describe the broadband frequency behavior of microwave systems, since, at microwave frequencies the $\mathbf{Y}$-representation cannot be accurately measured because the required short-circuit tests are difficult to achieve over a broad range of frequencies. Consequently, a transformation from $\mathbf{Y}$-representation into $\mathbf{S}$-representation is performed, while preserving stability and passivity by the procedure described in [20]. We recall that the bounded-realness property represents the passivity property for systems described by scattering parameters [17]. A system $\mathbf{S}(s)$ is bounded-real [17] if

1. $\mathbf{S}\left(s^{*}\right)=\mathbf{S}^{*}(s)$ for all $s$, where " $*$ " is the complex conjugate operator.

2. $\mathbf{S}(s)$ is analytic in $\Re e(s)>0$.

3. $\mathbf{I}-\mathbf{S}^{T}\left(s^{*}\right) \mathbf{S}(s) \geq 0 ; \forall s: \Re e(s)>0$.

In this paper we refer to these initial $\mathbf{S}$-reduced order models as root ROMs. The construction of the root ROMs results in a family of stable and passive univariate reduced models related to a specific set of points in the design space. The design space $\mathcal{D}(\boldsymbol{g})$ is defined as the parameter space $\mathcal{P}(s, \boldsymbol{g})$ without frequency. The parameter space $\mathcal{P}(s, \boldsymbol{g})$ contains all parameters $(s, \boldsymbol{g})$. If the parameter space is N-dimensional, the design space is (N-1)-dimensional.

3.2 2-D PMOR First, we discuss the representation of a bivariate reduced order model and afterwards the generalization to more dimensions. Once the root ROMs are available, the next step is to find a bivariate reduced model $\mathbf{S}_{r}(s, g)$ that preserves stability and passivity over the entire design space. The bivariate reduced model we adopt can be written as

$$
\mathbf{S}_{r}(s, g)=\sum_{k=1}^{K_{1}} \mathbf{S}_{r}\left(s, g_{k}\right) \ell_{k}(g)
$$

where $K_{1}$ is the number of the root ROMs, and the interpolation kernels $\ell_{k}(g)$ are scalar functions satisfying the following constraints

$$
\begin{aligned}
& 0 \leq \ell_{k}(g) \leq 1, \\
& \ell_{k}\left(g_{i}\right)=\delta_{k, i}, \\
& \sum_{k=1}^{K_{1}} \ell_{k}(g)=1 .
\end{aligned}
$$

A suitable choice is to select the set $\ell_{k}(g)$ as in piecewise linear interpolation. The reduced model in (3) is a linear combination of stable and passive univariate reduced models by means of a class of positive interpolation kernels [11]. Stability is automatically preserved in (3), since it is a weighted sum of stable rational models of $s$. The proof of the passivity-preserving property of the proposed PMOR scheme over the entire design space is given in Section 3.4.

3.3 (N+1)-D PMOR The bivariate formulation (3) can be easily generalized to the multivariate case by using multivariate interpolation methods, e.g. by means of tensor product [21]. The parametric reduced model can be written as

$$
\begin{aligned}
& \mathbf{S}_{r}(s, \boldsymbol{g})= \\
& =\sum_{k_{1}=1}^{K_{1}} \cdots \sum_{k_{N}=1}^{K_{N}} \mathbf{S}_{r}\left(s, g_{k_{1}}^{(1)}, \ldots, g_{k_{N}}^{(\mathrm{N})}\right) \ell_{k_{1}}\left(g^{(1)}\right) \cdots \ell_{k_{N}}\left(g^{(\mathrm{N})}\right)
\end{aligned}
$$


where $\ell_{k_{i}}\left(g^{(i)}\right), i=1, \ldots, \mathrm{N}$ satisfy all constraints (4)-(6). A suitable choice is to select each set $\ell_{k_{i}}\left(g^{(i)}\right)$ as in piecewise linear interpolation, which yields to an interpolation scheme in (7) called piecewise multilinear. This multivariate interpolation method can be also seen as a recursive implementation of 1-D piecewise linear interpolation. We remark that the interpolation process is local, because the parametric reduced model $\mathbf{S}_{r}(s, \boldsymbol{g})$ at a specific point $\widehat{\boldsymbol{g}}$ in the design space $\mathcal{D}(\boldsymbol{g})$ only depends on the root ROMs at the vertices of the hypercube that contains the point $\widehat{\boldsymbol{g}}$. An hypercube in $\mathbb{R}^{\mathrm{N}}$ has $2^{\mathrm{N}}$ vertices, $2^{\mathrm{N}}$ increases exponentially with the number of dimensions, but it still remains much smaller than the number of data points $K_{1} \cdot K_{2} \cdot \ldots \cdot K_{\mathrm{N}}$ in the fully filled design space grid. This multivariate interpolation method belongs to the general class of positive interpolation schemes [11]. We note that the interpolation kernels we propose only depend on the design space grid points and their computation does not require the solution of a linear system to impose an interpolation constraint.

The proposed PMOR technique is general and any interpolation scheme that leads to a parametric reduced model composed of a weighted sum of root ROMs with weights satisfying (4)-(6) can be used.

3.4 Passivity-Preserving Interpolation In this section, we prove that the proposed PMOR method preserves passivity (bounded-realness) over the entire design space. Concerning the root ROMs, they are bounded-real by construction. Condition 1) is preserved in (3) and the proposed multivariate extension (7), since they are weighted sums with real nonnegative weights of systems respecting this first condition. Condition 2 ) is preserved in (3),(7), since they are weighted sums of stable rational reduced models of $s$. Condition 3 ) is equivalent to $\|\mathbf{S}(s)\|_{\infty} \leq 1\left(\mathbf{H}_{\infty}\right.$ norm) [22], i.e., the largest singular value of $\mathbf{S}(s)$ does not exceed one in the right-half $s$-plane. Using this equivalent condition, in the bivariate case we can write

$$
\left\|\mathbf{S}_{r}(s, g)\right\|_{\infty} \leq \sum_{k=1}^{K_{1}}\left\|\mathbf{S}_{r}\left(s, g_{k}\right)\right\|_{\infty} \ell_{k}(g) \leq \sum_{k=1}^{K_{1}} \ell_{k}(g)=1
$$

Similar results are obtained for the proposed multivariate case (7), so condition 3) is satisfied by construction using our PMOR method. We have demonstrated that all three bounded-realness conditions are preserved in the novel PMOR algorithm, using the sufficient conditions (4)-(6) related to the interpolation kernels.

\section{Numerical example}

In this example a microstrip line with a dispersive DriClad dielectric $\varepsilon_{r}=4.1$ and a length $\ell=2 \mathrm{~cm}$ has been modeled. Its cross section is shown in Fig. 2. The dielectric and conductor thickness values are $h=600 \mu \mathrm{m}$ and $t=100 \mu \mathrm{m}$, respectively. A bivariate reduced order model is built as a function of frequency and the width of the strip $W$. Their corresponding ranges are freq $\in\left[1 \cdot 10^{3}-4 \cdot 10^{9}\right] \mathrm{Hz}$ and $W \in[50-250] \mu \mathrm{m}$, respectively.

The PEEC method is used to compute the $\mathbf{C}, \mathbf{G}, \mathbf{B}, \mathbf{L}$ matrices in (1a)-(1b) for 30 values of the width. The order of all

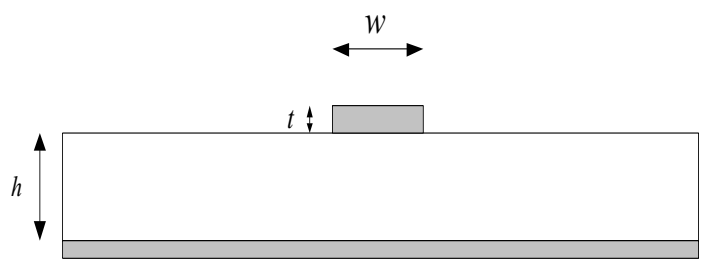

Figure 2: Cross section of the microstrip.

original PEEC models is equal to $n_{u}=2532$. Then, reduced models have been built for 12 values of the width by means of the Laguerre-based MOR algorithm, each with a reduced order $q=20$. A $\mathbf{Y}-\mathbf{S}$ transformation has been performed choosing $\mathrm{Z}_{0,1}=\mathrm{Z}_{0,2}=50 \Omega$, which results in a set of 12 root ROMs. A bivariate reduced model $\mathbf{S}_{r}(s, W)$ is obtained by piecewise linear interpolation of the root ROMs. Fig. 3 shows the magnitude of the parametric reduced model of $\mathbf{S}_{11}(s, W)$. Fig. 4 shows the magnitude of the parametric reduced model of $\mathbf{S}_{21}(s, W)$ for the width values $W=\{55,150,245\} \mu \mathrm{m}$. These specific width values have not been used in the root ROM generation process, nevertheless an excellent agreement between model and data can be observed. The maximum absolute error of the bivariate reduced model of the $\mathbf{S}$ matrix over a dense reference grid composed of $200 \times 30($ freq, $W$ ) samples is bounded by $-60.57 \mathrm{~dB}$. As clearly seen, the parametric reduced model captures very accurately the behavior of the system, while guaranteeing stability and passivity properties over the entire design space.

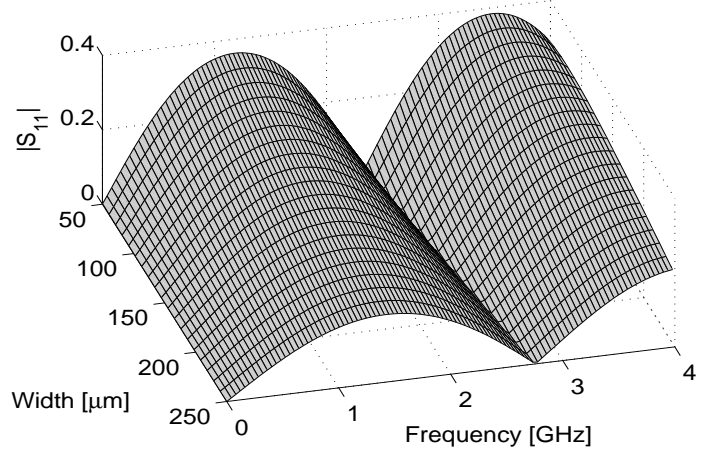

Figure 3: Magnitude of the bivariate reduced model of $\mathbf{S}_{11}(s, W)$.

\section{Conclusions}

We have presented a new parameterized model order reduction technique applicable to PEEC analysis. The overall stability and passivity of the parametric reduced order model is guaranteed by an efficient and reliable combination of traditional passivity-preserving MOR methods and positive interpolation schemes. The numerical results show the capability of the proposed PMOR approach of generating very accurate parametric reduced models, while guaranteeing stability and passivity over the entire design space of interest. 


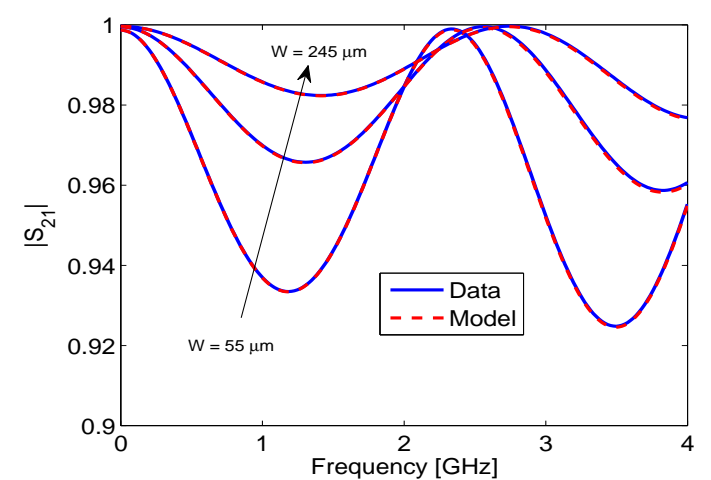

Figure 4: Magnitude of the bivariate reduced model of $\mathbf{S}_{21}(s, W)(W=\{55,150,245\} \mu \mathrm{m})$.

\section{Acknowledgements}

This work was supported by the Research Foundation Flanders (FWO).

\section{References}

[1] R. F. Harrington, Field Computation by Moment Methods. New York: Macmillan, 1968.

[2] A. E. Ruehli, "Equivalent circuit models for three dimensional multiconductor systems," IEEE Trans. Microw. Theory Tech., vol. 22, no. 3, pp. 216-221, Mar. 1974.

[3] J. M. Jin, The Finite Element Method in Electromagnetics, 2nd ed. John Wiley and Sons, New York, 2002.

[4] A. Odabasioglu, M. Celik, and L. T. Pileggi, "PRIMA: passive reduced-order interconnect macromodeling algorithm," IEEE Trans. Comput.-Aided Design Integr. Circuits Syst., vol. 17, no. 8, pp. 645-654, Aug. 1998.

[5] L. Knockaert and D. De Zutter, "Passive reduced order multiport modeling: the Padé-Laguerre, Krylov-ArnoldiSVD connection," Int. J. Electron. Commun. (AË̈), vol. 53, no. 5, pp. 254-260, 1999.

[6] A. E. Ruehli and A. C. Cangellaris, "Progress in the methodologies for the electrical modeling of interconnects and electronic packages," Proc. IEEE, vol. 89, no. 5, pp. 740-771, May 2001.

[7] P. K. Gunupudi, R. Khazaka, M. S. Nakhla, T. Smy, and D. Celo, "Passive parameterized time-domain macromodels for high-speed transmission-line networks," IEEE Trans. Microw. Theory Tech., vol. 51, no. 12, pp. 23472354, Dec. 2003.

[8] L. Daniel, O. C. Siong, L. S. Chay, K. H. Lee, and J. White, "A multiparameter moment-matching modelreduction approach for generating geometrically parameterized interconnect performance models," IEEE Trans. Comput.-Aided Design Integr. Circuits Syst., vol. 23, no. 5, pp. 678-693, May 2004.
[9] P. Heydari and M. Pedram, "Model reduction of variablegeometry interconnects using variational spectrallyweighted balanced truncation," in Proc. IEEE/ACM Int. Conf. Comput.-Aided Des., Nov. 4-8, 2001, pp. 586-591.

[10] J. R. Phillips, "Variational interconnect analysis via PMTBR," in Proc. IEEE/ACM Int. Conf. Comput.-Aided Des., Nov. 7-11, 2004, pp. 872-879.

[11] G. Allasia, "Simultaneous interpolation and approximation by a class of multivariate positive operators," Numerical Algorithms, vol. 34, no. 2, pp. 147-158, Dec. 2003.

[12] F. Ferranti, L. Knockaert, and T. Dhaene, "Parameterized S-parameter based macromodeling with guaranteed passivity," IEEE Microw. Wireless Compon. Lett., vol. 19, no. 10, pp. 608-610, Oct. 2009.

[13] G. Wollenberg, A. Görisch, "Analysis of 3-D interconnect structures with PEEC using SPICE," IEEE Trans. Electromagn. Compat., vol. 41, no. 2, pp. 412-417, Nov. 1999.

[14] A. E. Ruehli, G. Antonini, J. Esch, J. Ekman, A. Mayo and A. Orlandi, "Non-orthogonal PEEC formulation for time and frequency domain EM and circuit modeling," IEEE Trans. Electromagn. Compat., vol. 45, no. 2, pp. 167-176, May 2003.

[15] C. Ho, A. Ruehli, P. Brennan, "The modified nodal approach to network analysis," IEEE Trans. Circuits Syst., vol. 22, no. 6, pp. 504-509, June 1975.

[16] R. Rohrer, H. Nosrati, "Passivity considerations in stability studies of numerical integration algorithms," IEEE Trans. Circuits Syst., no. 9, pp. 857-866, Sept. 1981.

[17] B. D. Anderson, S. Vongpanitlerd, Network Analysis and Synthesis. Englewood Cliffs, NJ, 1973.

[18] D. D. Ling and A. E. Ruehli, Circuit Analysis, Simulation and Design, 2. Elsevier Science Publishers B. V. (NorthHolland), 1987, pp. 211-253.

[19] R. W. Freund, "Krylov-subspace methods for reducedorder modeling in circuit simulation," J. Comput. Appl. Math., vol. 123, no. 1-2, pp. 395-421, 2000.

[20] C. P. Coelho, J. R. Phillips, and L. M. Silveira, "Passive constrained rational approximation algorithm using Nevanlinna-Pick interpolation," in Proc. Design, Automation and Test in Europe Conference and Exhibition, Mar. 4-8, 2002, pp. 923-930.

[21] E. W. Cheney, "Multivariate approximation theory: Selected topics," in CBMS-NSF Regional Conference Series in Applied Mathematics, vol. 51. Philadelphia, PA: SIAM, 1986.

[22] S. Boyd, L. El Ghaoui, E. Feron and V. Balakrishnan, Linear Matrix Inequalities in System and Control Theory. Philadelphia: SIAM, 1994. 